\title{
FRAGMENTACION DEL PAISAJE DE LA CUENCA DEL RÍO LIMOY
}

\author{
André Luis Sotille Riedi \\ andre_sotile@hotmail.com \\ Universidad Nacional Del Este \\ Facultad De Ingeniería Agronómica \\ Carrera De Ingeniería Ambiental \\ Minga Guazú- Paraguay
}




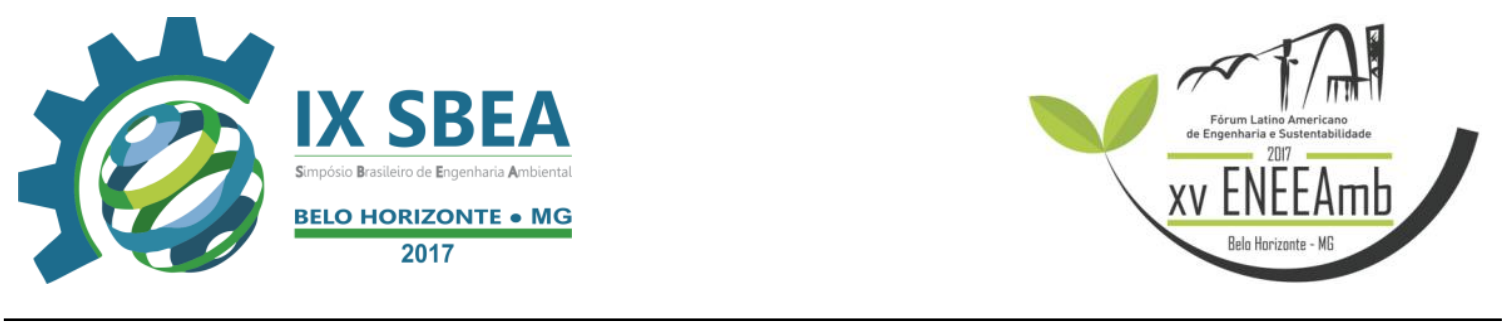

\section{RESUMO}

El presente trabajo estudió la fragmentación del paisaje de la cuenca del Río Limoy, mediante la utilización de herramientas de teledetección y sistema de información geográfico, periodo comprendido de 1985 a 2015(30 años). Primeramente, se realizó un análisis multitemporal de la variación de la cobertura forestal de la cuenca, todos los datos fueron procesados y analizados mediante la plataforma ArcGis y extensiones para la misma. Mediante el análisis multitemporal fue posible determinar índices de cuantificación formal de la cuenca. Una vez obtenidos los índices de cuantificación formal, fue posible calcular los índices de forma y densidad que permitieron estimar la heterogeneidad de la cuenca, así también de este modo fue posible calcular el grado de conectividad de los fragmentos de la cobertura forestal de la cuenca, permitiendo de este modo, mediante la elaboración de mapas temáticos dar una descripción espacial de su grado de fragmentación. Mediante los análisis se determinó una disminución del 74\% de la cobertura forestal en treinta años; produciéndose así una fragmentación de carácter heterogéneo con áreas nucleares pequeñas con una distribución desproporcional y conectividad nula desde 1995.

Palabras claves: Fragmentación de paisaje, ladscape metrics, cobertura forestal, teledetección

\section{INTRODUÇÃO/OBJETIVO}

Gran parte de la superficie terrestre está siendo afectada por actividades humanas, ya sea mediante la extracción de recursos o a través de la transformación de los ecosistemas naturales en otros dominados por usos antrópicos. Sanderson (2002) afirma que los patrones de uso y cambios en el uso de la tierra tienen estrecha relación tanto con las características físicas y ecológicas del paisaje, como con las condiciones de desarrollo socioeconómico y político de una región.

Los cambios de cobertura e índice de fragmentación de la cobertura forestal constituyen información cuantitativa y cualitativa importante, que una vez representadas en mapas y datos estadísticos, servirán como una herramienta valiosa para plantear proyectos de conservación en las zonas más intervenidas.

La Cuenca del Río Limoy se encuentra dentro de la Ecorregión Bosque Atlántico del Alto Paraná, según la ecorregión del Bosque Atlántico del Alto Paraná es 


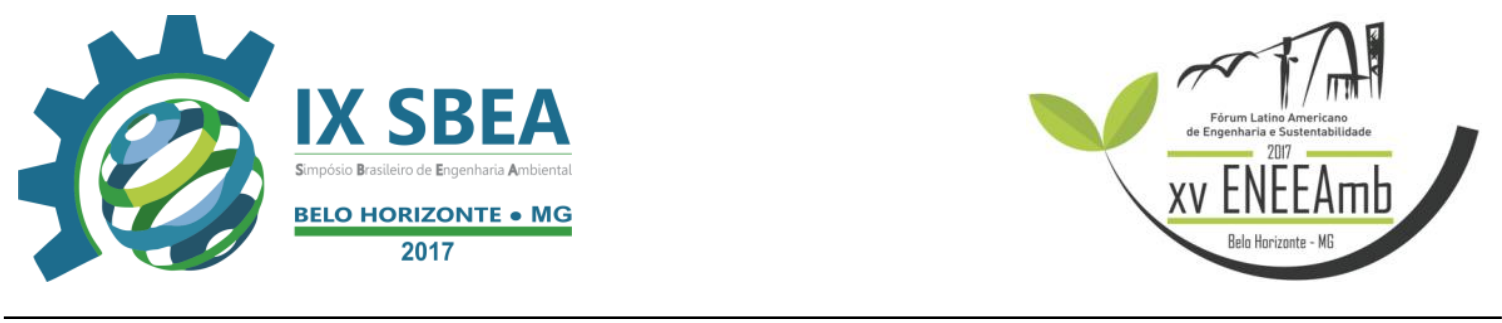

un caso extremo de la capacidad del hombre de modificar ambientes naturales. Entonces desde ese punto de vista conocer los patrones de cambios de la cobertura forestal, su grado de fragmentación y sus vínculos con los conductores socio-económicos e impactos ambientales, son esenciales para la planificación y discusión de las vías y políticas de desarrollo para la conservación, conexión y o recuperación. Esto nos permite caracterizar un paisaje no solo en su configuración actual, sino en su historia y dinámica, lo que es fundamental para comprender las interacciones entre el paisaje y la biodiversidad, y predecir estados futuros.

Otro punto importante a tener en cuenta es que dentro de los límites de la Cuenca del Río Limoy se encuentra la Reserva Biológica de Limoy, lo que enfatiza la importancia de la utilización de herramientas adecuadas para medir los índices de fragmentación del paisaje con el fin de establecer medidas de conservación.

Uno de los métodos de medir la fragmentación del paisaje es la utilización de sensores remotos y sistemas de información geográfica. Tal herramienta se encuentra cada vez más extendido dentro de la biología de la conservación, ya que facilita el desarrollo de proyectos en zonas poco accesibles, disminuye su tiempo de elaboración y los resultados obtenidos concuerdan con los objetivos planteados.

El presente estudio determinó los cambios de cobertura forestal en la zona entre los años 1985 a 2015, utilizando distintos métodos y técnicas de análisis multitemporal, con imágenes satelitales. Además, se aplicaró técnicas de medición del paisaje con el fin de identificar el índice de fragmentación para el año 2015.

\section{OBJETIVOS}

\section{GENERAL}

- Determinar el nivel de fragmentación del paisaje (Landscape metrics) de la Cuenca del Río Limoy a través de herramientas de teledetección e información geográfica (SIG), en periodo de 1985 al 2015.

\section{ESPECÍFICOS}

- Analizar la variación de la cobertura forestal de la Cuenca del Río Limoy.

- Cuantificar a los fragmentos de la cobertura forestal de la Cuenca del Río Limoy en índices de cuantificación formal. 


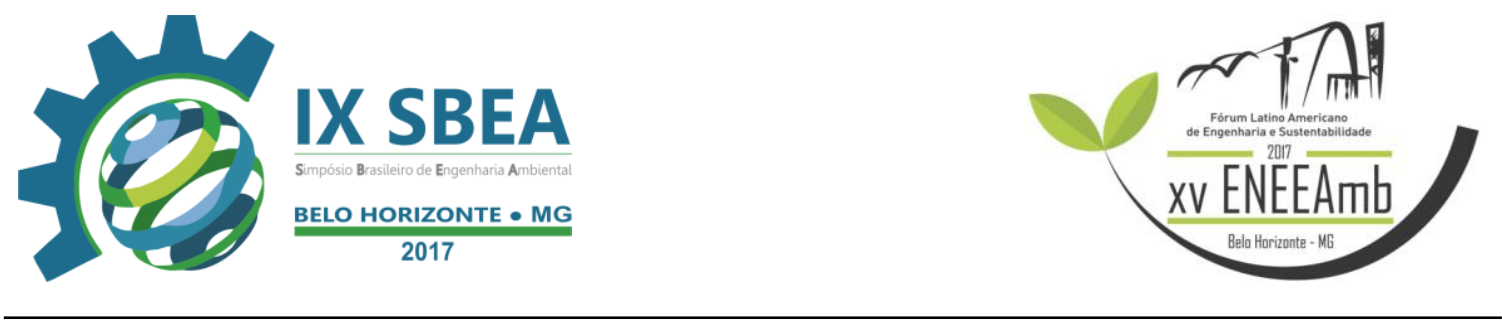

- Determinar la conectividad de los fragmentos de la cobertura forestal Cuenca del Río Limoy.

Determinar la heterogeneidad o homogeneidad de la cobertura forestal de la Cuenca del Río Limoy

\section{METODOLOGIA}

\section{Localización}

El área de estudio comprende a la Cuenca del Río Limoy; posee una superficie de 1.058 kilómetros cuadrados. La cuenca está ubicada en la Región Oriental del Paraguay, se encuentra dentro del Departamento de Alto Paraná, margen derecha del Río Paraná, a $160 \mathrm{Km}$. al norte de la cuidad de Hernandarias; forman parte de la cuenca los distritos de Itakyry, Mbaracayu, Minga Pora y San Alberto.

\section{Diseño de Investigación}

El tipo de investigación pertenece al estudio descriptivo no experimental con diseño longitudinal.

Según Hernandez R.; Fernández C.; Baptista P., (2003) con mucha frecuencia, el propósito del investigador consiste en describir situaciones, eventos y hechos. Esto es, decir como es y cómo se manifiesta determinado fenómeno. Los estudios descriptivos buscan especificar las propiedades, las características y los perfiles importantes de personas, grupos, comunidades o cualquier otro fenómeno que se someta a un análisis. Los diseños longitudinales recolectan datos sobre variables o sus relaciones, en dos o más momentos, para evaluar el cambio en estas.

\section{Selección de Imágenes y Procesamiento}

Para evaluar la variación de lo cobertura forestal de la cuenca del Río Limoy, se realizó un análisis multitemporal, a través de imagines Landsat, de los años; 1985, 1995, 2005 y 2015. Las imágenes fueron tratadas y procesadas en la plataforma Arc gis.La clasificación se imágenes satelitales se realizó mediante el proceso de clasificación nosupervisada con el algoritmo ISO-Cluster. Se realizó la delimitación de la cuenca del Río Limoy utilizando el modelo digital del terreno (MDT) del Space Radar Shuttle (SRTM) con una resolución espacial de 30m. Mediante las herramientas de hidrología disponibles en el ArcGIS 10.2.2. Las imágenes se descargaron del servidor de Imágenes GLOVIS 


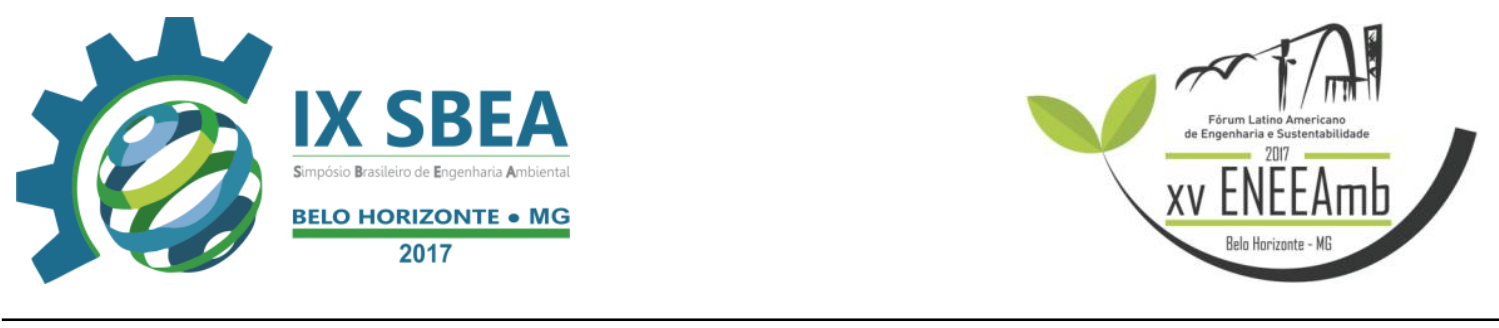

\section{Índices de ecología del paisaje.}

Obtención de las métricas de ecología del paisaje.

Para realizar la medición en índices de cuantificación formal primeramente se realizó un análisis multitemporal de la modificación de la cobertura forestal de la Cuenca del Río Limoy, de este modo se delimito los fragmentos en índices de cuantificación formal, una vez obtenidos los índices de cuantificación formal se procedió al análisis de los índices de conectividad. La conectividad de los fragmentos de la cobertura forestal se determinó a través de las características de los índices de configuración del paisaje McGarigal (2002). Por último, se determinó la homogeneidad y o heterogeneidad del paisaje utilizando índices de forma y densidad del paisaje.

\section{RESULTADOS E DISCUSSÃO}

Mediante los resultados obtenidos en la determinación de la fragmentación del paisaje de la Cuenca del Río Limoy atreves de la utilización de herramientas de teledetección e información geográfica, observase que hubo a lo largo de 30 años grandes cambios en la estructura del paisaje de la cuenca. Estos cambios provocaron gradualmente la disminución de la cobertura forestal conduciendo a un grado de fragmentación extremo. Con los resultados obtenidos primeramente se realizó un análisis de la variación de la cobertura forestal, seguidamente se determinaron índices de cuantificación formal que permitieron analizar el grado de conectividad y homogeneidad de la cuenca a lo largo del periodo de estudio.

\section{Análisis Multitemporal}

Para determinar la variación de cobertura forestal de la cuenca del Río Limoy se procedió a la tabulación cruzada de resultados obtenidos en el procesamiento de las imágenes lansat mediante la plataforma de Arc Gis 10.2.2. La figura 11 demuestra respectivamente la variación de la cobertura forestal durante los años 1985 a 2015. Mediante el análisis de tabulación cruzada, observase que durante la primera década (1985 a 1995), hubo una disminución de la cobertura forestal de 42566 ha. En el año 1985 la cuenca poseía una cobertura forestal de 61585 ha, lo que corresponde a una pérdida de 69\% de la cobertura en una década. Para la década siguiente (1995 a 2005) se observa una disminución de 9323ha, en relación a 2005. 


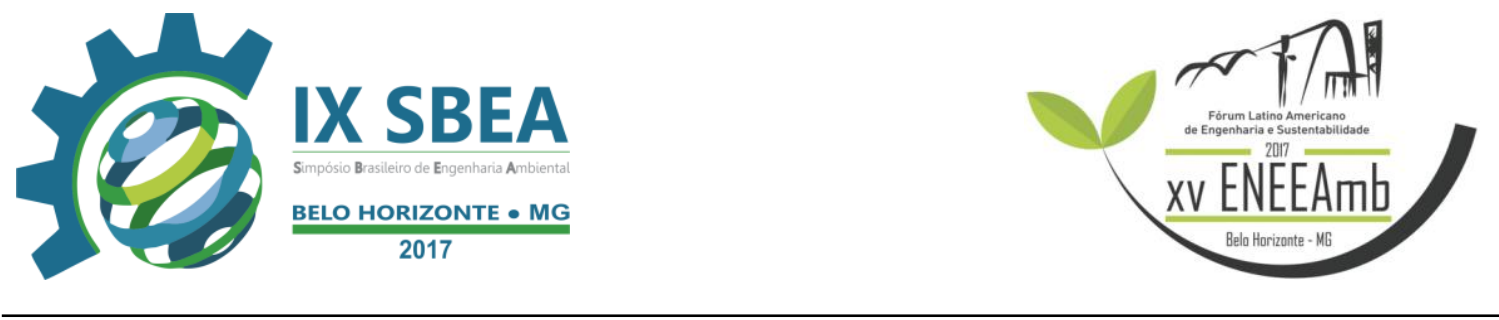

Figura 11- Variación de Cobertura Forestal en periodo de 1985 a 2015

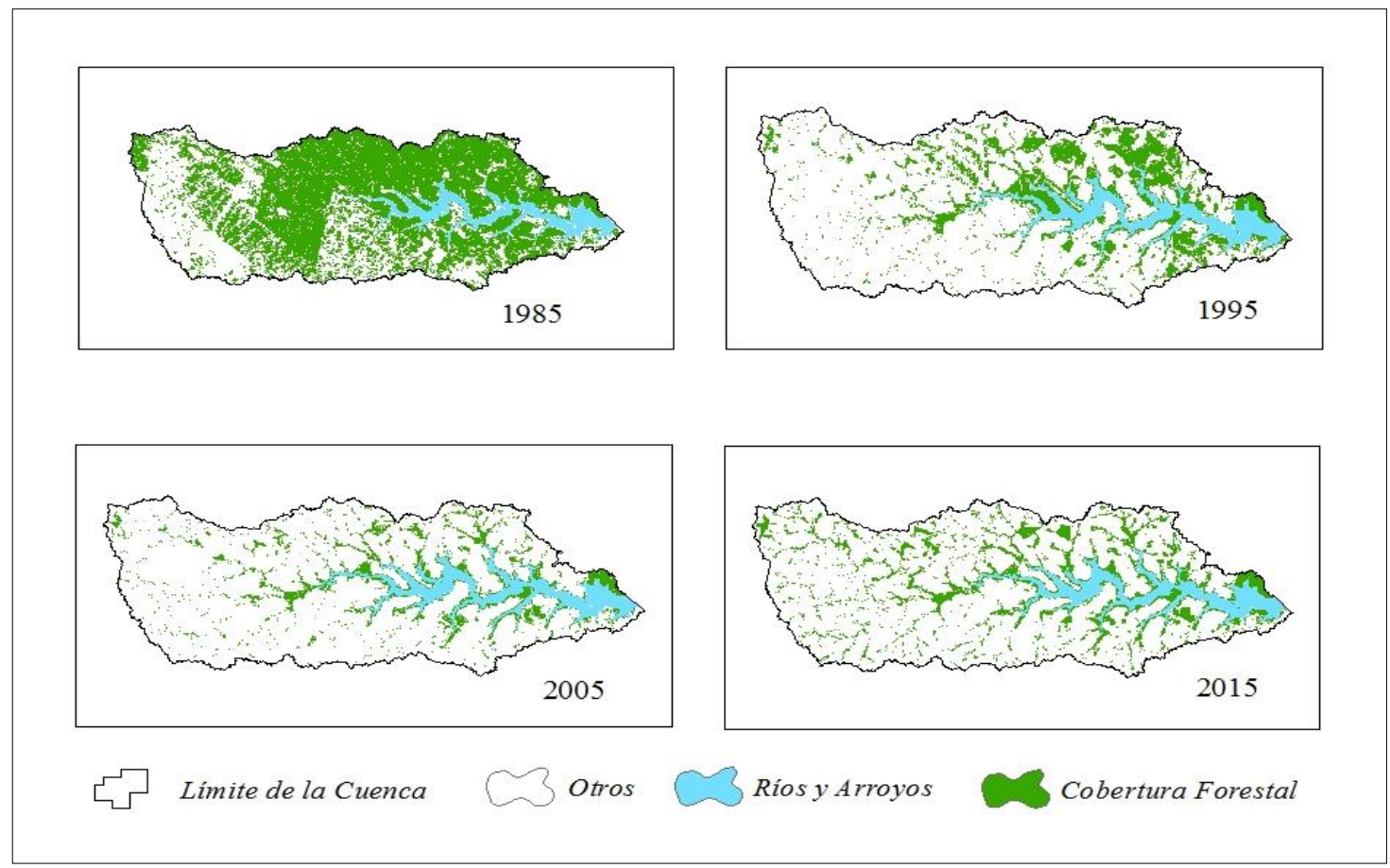

Fuente: Elaboración propia. Minga Guazú 2016

La última década de estudio (2005 a 2015) se registra un aumento de 5735 ha. de la cobertura forestal en la cuenca del Río Limoy. Este dato podría explicarse de la siguiente manera:A finales del año 2004 se promulga la Ley 2524/04, "De Prohibición En La Región Oriental De Las Actividades De Transformación Y Conversión De Superficies Con Cobertura De Bosques", provocando de esta manera la disminución de la tala y quema ilegal de bosque nativos.

Por otro lado, el aumento de la cobertura forestal durante esta década podría explicarse con la consolidación de la Secretaria del Medio Ambiente (SEAM) durante los años 2004 a 2008, y la efectiva aplicación de la Ley 294/93 de "Evaluación De Impacto Ambiental".

A pesar de que la Ley estaba en vigencia desde 1993, su aplicación según Molinas (2013) durante este periodo de administración de este marco legal se sustentó en la aplicación de los mismos para las grandes obras especialmente las financiadas por organismos internacionales como el Banco Mundial (BM) y el Banco Interamericano de Desarrollo (BID), donde prácticamente sus préstamos aprobados por el parlamento para el país especialmente destinados su ejecución al Ministerio de Obras Públicas y Comunicaciones (MOPC).

La puesta en vigencia del Decreto $N^{o} \mathbf{1 4 . 2 8 1 / 9 6}$ que reglamenta la Ley $N^{o} 294 / 93$ de Evaluación de Impacto Ambiental, después de casi cuatro años de estar vigente la ley se establecía claramente los términos, normas técnicas y procedimientos administrativos de la 


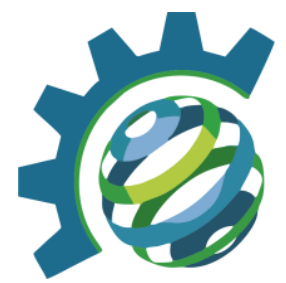

Evaluación de Impacto Ambiental en el Paraguay. Aún según Molinas (2013), en el periodo de 1.996 al 2.000 se crea la Secretaria del Ambiente (SEAM) dependiente del Ministerio de Agricultura y Ganadería (MAG), y se convierte en la autoridad administrativa de la Ley $\boldsymbol{N}^{\boldsymbol{o}}$ 294/93 de Evaluación de Impacto Ambiental y su Decreto Nº14.281/96 en el Paraguay. Pero en el periodo del 2000 al 2003 y parte del 2004 la Evaluación de Impacto ambiental en el Paraguay sufre una retracción en el proceso de consolidación, debido a que la Secretaria del Ambiente (SEAM) entra en una serie de cambios sucesivos de sus ministros secretarios y asociado además al cambio de gobierno, donde el tema ambiental en el país se debilita como política y finalmente repercute en la consolidación de la Evaluación de Impacto Ambiental aflorando principalmente los problemas de extorción y corrupción como principales elementos en el proceso en la Evaluación de Impacto Ambiental debilitando su administración y aplicación en este proceso.

Finalmente, en el periodo del 2004 al 2008 la Secretaria del Ambiente (SEAM) se estabiliza logrando un fuerte liderazgo con el apoyo financiero del Banco Interamericano de Desarrollo (BID), donde se formula, ajusta y promulga la Política Ambiental Nacional (PAN/2005) convirtiendo a la Evaluación de Impacto Ambiental en el principal instrumento u herramienta de gestión ambiental en el Paraguay.

Tomando en cuenta el periodo de estudio de 1985 a 2015, (30 años), se observa según la figura 12, una disminución de la cobertura forestal de 46154 ha, se afirma así, que hubo una disminución de $74 \%$ de la cobertura forestal en la Cuenca del Río Limoy durante los últimos 30 años.

Figura 12- Superposición de imágenes de la Cobertura Forestal de la Cuenca del Río Limoy entre los años 1985 y 2015

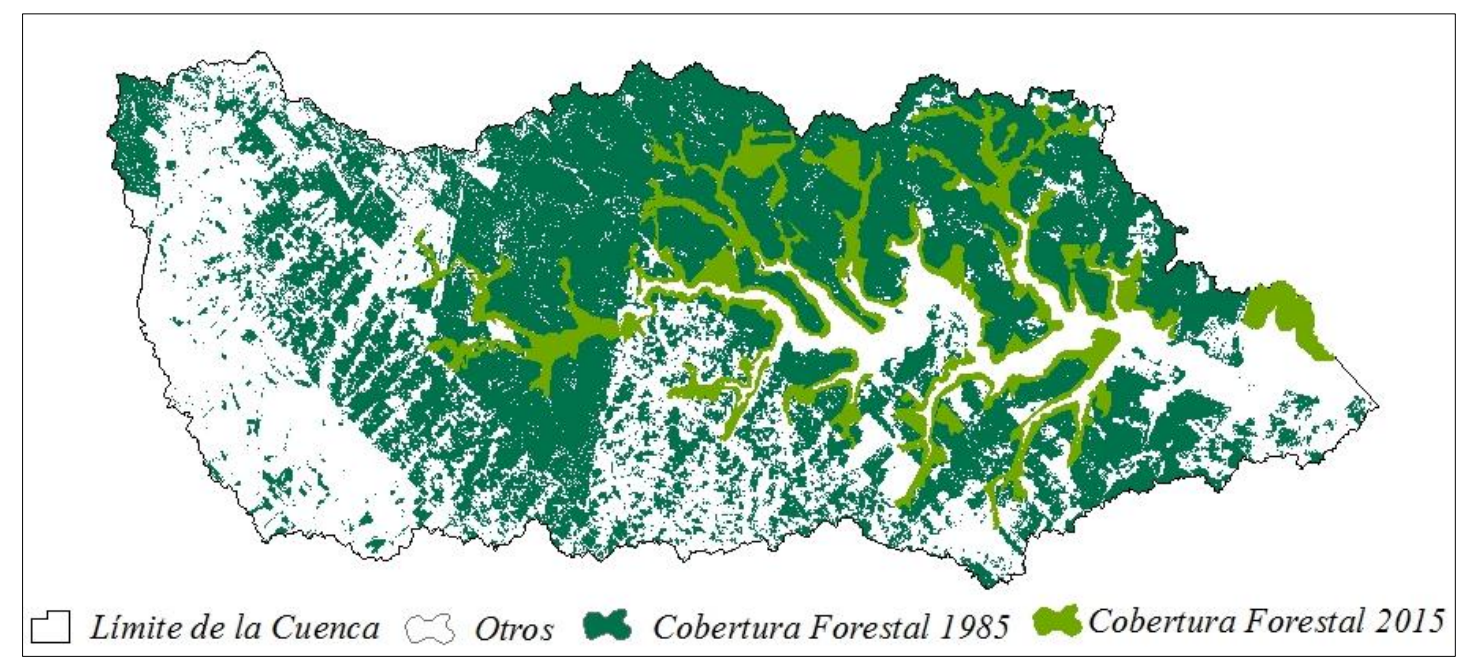

Fuente: Elaboración propia. Minga Guazú. 2016 


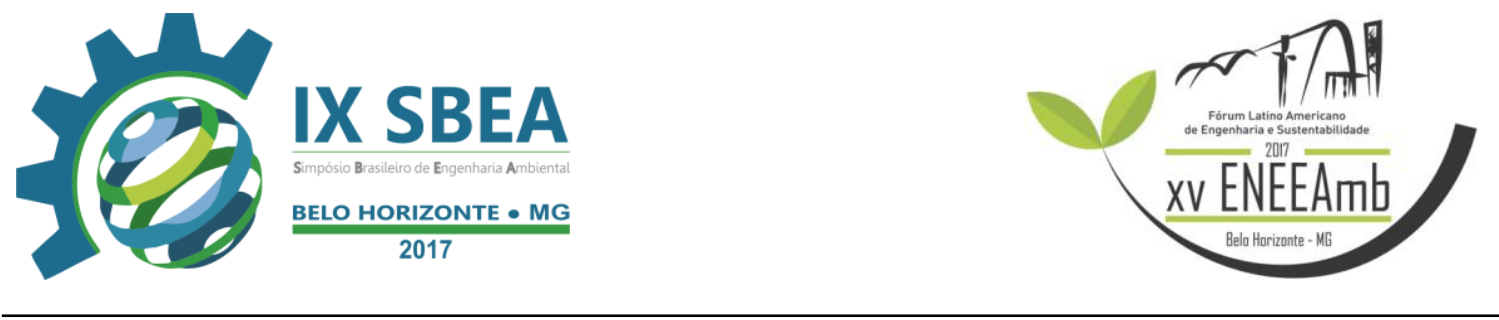

En la figura 13 se observa las áreas nucleares consideradas matrices, las mismas fueron indicadas por la extensión para la plataforma Arc Gis, denominada Patch Analyst mediante los datos cargados, donde se aplicaron los cálculos y métricas utilizadas por Olivier (2013) para calcular el grado de conectividad, el mismo autor tomo como base los índices de proximidad, CONECT cuando el Índice de Proximidad Medio es menor a 1000m, media cuando es menor a $2000 \mathrm{~m}$, baja mayor a $2000 \mathrm{~m}$ y nula mayor a $5000 \mathrm{~m}$.

Figura 13- Áreas Nucleares Matrices de la Cuenca del Río Limoy en periodo de 1985 a 2015

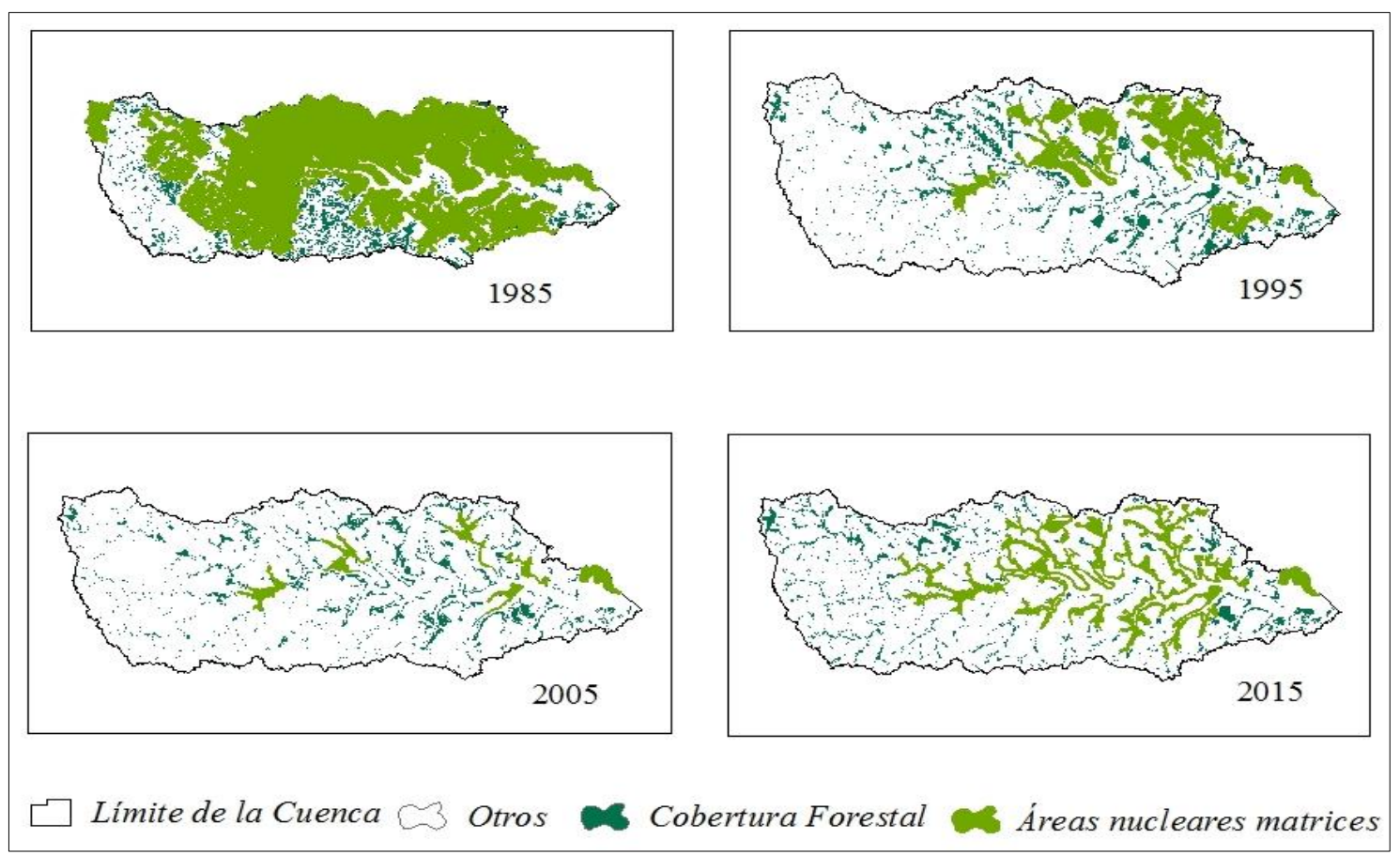

Fuente: Elaboración propia. Minga Guazú. 2016

De esta manera se afirma que el paisaje de la cobertura forestal de la Cuenca del Río Limoy posee una conectividad Nula desde el año 1995, pero que para el año 2015 se registra una aproximación considerable para lograr la conectividad de los fragmentos a pesar de que las áreas nucleares consideradas matrices son relativamente pequeñas.

Cabe destacar que la importancia de la conectividad ecología en la cuenca del Río Limoy no solo se enfatiza a la conservación de las especies de flora presentes en la cuenca, sinembargo también a las especies faunísticas, ya que estas en cierta medida, existiendo un grado de conexión, serán responsables por garantizar procesos de migración, relación reproducción e intercambio genético. 


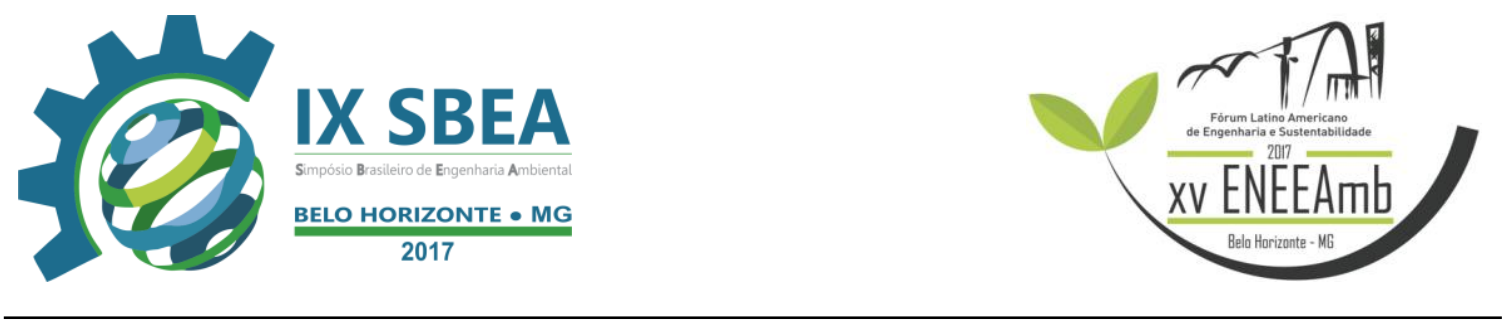

Lógicamente, cada especie contará con unos niveles de desplazamiento que serán distintos y que, por tanto, estarán condicionadas a las necesidades de conexión entre poblaciones de su misma especie, para así poder cumplir con su nicho ecológico y de este modo mantener un grado de equilibrio en la cuenca.

Finalmente se destaca que la importancia de esta investigación radica en que atreves del estudio de los efectos recíprocos entre el patrón espacial y los procesos ecológicos que se manifiestan a escala de paisaje; pueda establecerse métodos de conservación y o recuperación eficaces y efectivos. Además, esta investigación podrá auxiliar con información cualitativa y cuantitativa importante para la eficacia de programas nacionales de conservación y recuperación de la biodiversidad ya existentes en el país.

\section{CONCLUSÕES/RECOMENDAÇÕES}

Con base en los resultados obtenidos en los análisis realizados mediante herramientas de teledetección y sistema de información geográfica, en periodo de 1985 a 2015(30 años) en la Cuenca del Río Limoy, se presenta las siguientes conclusiones: En el análisis multitemporal, de la cobertura forestal de la cuenca del Río Limoy se determinó una pérdida de cobertura forestal de 46154 ha; se afirma así, que hubo una disminución de 74\% de la cobertura forestal en la Cuenca durante los últimos 30 años. Mediante el análisis de los índices de cuantificación formal se confirma que se ha producido en la cuenca atreves del tiempo una fragmentación considerable del paisaje con una reducción significativa del número de fragmentos, así como también el área total y el tamaño medio de los mismos. Con los valores obtenidos mediante los índices de forma y de diversidad del paisaje se afirma que para el año 2015 el paisaje de la Cuenca del Río Limoy es heterogéneo con fragmentos con áreas nucleares matrices relativamente pequeños y con una diversidad de distribución desproporcional. En los análisis de conectividad de los fragmentos de la cobertura forestal se observó que la conectividad de las áreas nucleares consideradas matrices en la cuenca es nula desde el año 1995. Finalmente cabe destacar que se registró un aumento de 5735 ha de la cobertura forestal en la última década, así como también los vales de proximidad se 


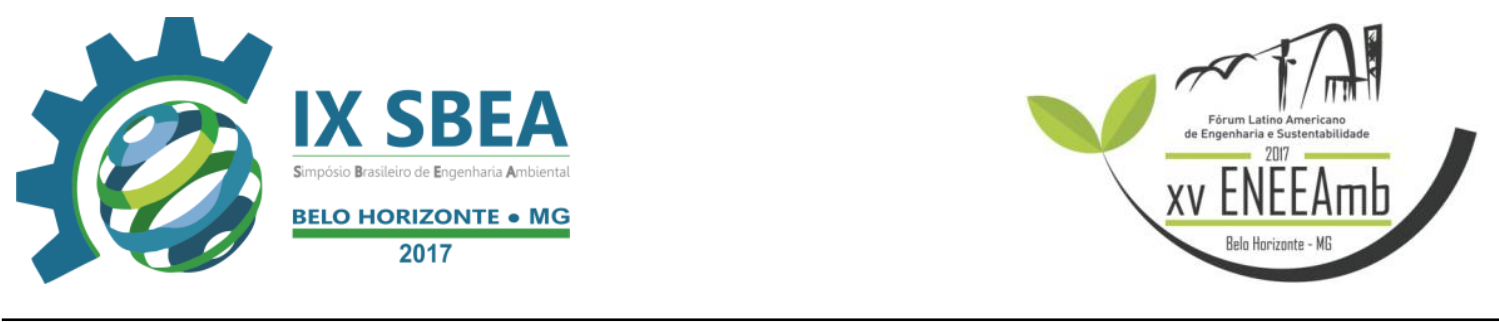

aproximan a índices considerados de conectividad, aunque para áreas nucleares matrices relativamente pequeñas.

\section{REFERÊNCIAS BIBLIOGRÁFICAS}

Acuña. Camilo 2010. Identificación De Áreas Prioritarias De Conservación Enfocadas Hacia La Conectividad Estructural Del Corredor Encenillo (Municipios De La Calera, Guasca, Sopo, Sesquilé, Guatavita), Cundinamarca. Pontificia Universidad Javeriana. Bogotá, Colombia.

Abajo, A (2007). Modelos espaciales de hábitad y análisis de viabilidad de la población de Urgallo en la Cordillera Cantábrica. Oviedo.

Etter .A. 2000. Introducción a la Ecología del Paisaje. Un Marco de Integración para los Levantamientos Rurales. Instituto Geográfico Agustín Codazzi, Subdirección de Docencia e Investigación, Unidad de Levantamientos Rurales. Bogotá, Colombia.

Fahrig, L. 2003. Effects of habitat fragmentation on biodiversity. Annu. Rev. Ecol. Evol. Syst.

Forman R.T.T. y Gordón, M. (1986): Landscape Ecology. New York, John Wiley and Sons.

Grossmann, W.D. (1993).Challenges from ecology to application and desig of Geographical Information System. Zaragoza.

Hernández R.; Fernández, C. y Baptista,(2003) "Metodología de la investigación" México D.F.: McGraw-Hill.

Morláns. María Cristina. 2013. Estructura Del Paisaje (Matriz, Parches, Bordes, Corredores) Sus Funciones Fragmentacion Del Habitat Y Su Efecto Borde. Editorial Científica Universitaria - Universidad Nacional de Catamarca.

Molinas. Alfredo. 2013. Medio Ambiente. Disponible en htt: www.ing-alfredomolinas.blogsport.com. Consultado el 11/02/20016.

McGarigal K, 2002 . FRAGSTATS version 3.3: Spatial Pattern Analysis Program for Categorical Maps, users manual. University of Massachusetts.

Olivier, F. Y Wotherspoon Sj. 2013. Modelling habitat selection using presence-only data : Case study of a colonial hollow nesting bird , the snow petrel. Ecological Modelling

Pontius Jr, Robert Gilmore, Emily Shusas and Menize McEachern. (2004). "Detecting impotant categorical land changes while accounting for persistence". Agriculture, Ecosystems \& Environment.

Sánchez Peña, Ramón Ovidio. 2006. Establecimiento y manejo de áreas protegidas: Notas básicas para la enseñanza. Instituto Tecnológico De Santo Domingo (INTEC). Santo Domingo.

Sanderson EW,Redford KH,Vedder A,Coppolillo PB,Ward SE. 2002. A conceptual model for conservation planning based on landscape species requirements. Landscape and Urban Planning. 


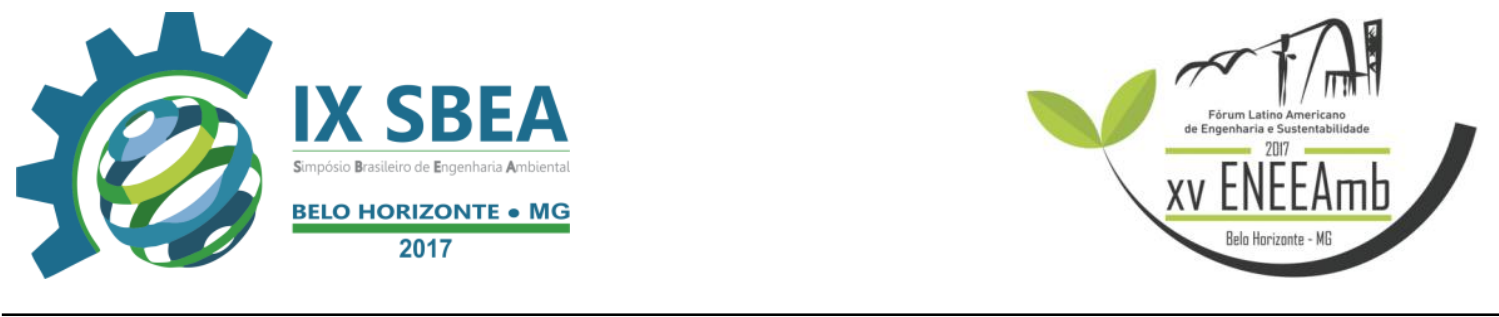

Taylor, G.D., y D. Lamb, redactores, 2006. La restauración ecológica: un medio para conservar la biodiversidad y mantener los medios de vida. Society for Ecological Restoration International, Tucson, Arizona, EE.UU. y IUCN, Gland, Suiza.

Villegas Florez, Eduardo. 2001. Análisis multitemporal de patrones especiales de transformación del paisaje (1940-1998) en una parte de los cerros orientales de Bogotá. Tésis (Ecólogo). Pontificia Universidad Javeriana.

Vila, J. D., Vargas, 2006. Conceptos y métodos fundamentales en ecología del paisaje (Landscape ecology). Una interpretación desde la geografía. Doc. Anal. Geogr .

Yerena, E. 2002. Corredores Ecológicos en los andes de Venezuela. Parques Nacionales y

Conservación Ambiental. Fundación Polar. Editorial Torino. Caracas, Venezuela. 